University of Nebraska - Lincoln

DigitalCommons@University of Nebraska - Lincoln

Faculty Publications from the Department of Electrical \& Computer Engineering, Department Electrical and Computer Engineering

2008

\title{
Analysis of Energy Efficiency in Fading Channels under QoS Constraints
}

Deli Qiao

University of Nebraska-Lincoln, dlqiao@ce.ecnu.edu.cn

Mustafa Cenk Gursoy

University of Nebraska-Lincoln, gursoy@engr.unl.edu

Senem Velipasalar

University of Nebraska-Lincoln, velipasa@engr.unl.edu

Follow this and additional works at: https://digitalcommons.unl.edu/electricalengineeringfacpub

Part of the Electrical and Computer Engineering Commons

Qiao, Deli; Gursoy, Mustafa Cenk; and Velipasalar, Senem, "Analysis of Energy Efficiency in Fading Channels under QoS Constraints" (2008). Faculty Publications from the Department of Electrical and Computer Engineering. 123.

https://digitalcommons.unl.edu/electricalengineeringfacpub/123

This Article is brought to you for free and open access by the Electrical \& Computer Engineering, Department of at DigitalCommons@University of Nebraska - Lincoln. It has been accepted for inclusion in Faculty Publications from the Department of Electrical and Computer Engineering by an authorized administrator of DigitalCommons@University of Nebraska - Lincoln. 


\title{
Analysis of Energy Efficiency in Fading Channels under QoS Constraints
}

\author{
Deli Qiao, Mustafa Cenk Gursoy, Senem Velipasalar \\ Department of Electrical Engineering \\ University of Nebraska-Lincoln, Lincoln, NE 68588 \\ Email: qd1726@bigred.unl.edu,gursoy@engr.unl.edu,velipasa@engr.unl.edu
}

\begin{abstract}
Energy efficiency in fading channels in the presence of QoS constraints is studied. Effective capacity, which provides the maximum constant arrival rate that a given process can support while satisfying statistical delay constraints, is considered. Spectral efficiency-bit energy tradeoff is analyzed in the low-power and wideband regimes by employing the effective capacity formulation, rather than the Shannon capacity, and energy requirements under $\mathrm{QoS}$ constraints are identified. The analysis is conducted for the case in which perfect channel side information (CSI) is available at the receiver and also for the case in which perfect CSI is available at both the receiver and transmitter. In particular, it is shown in the low-power regime that the minimum bit energy required in the presence of QoS constraints is the same as that attained when there are no such limitations. However, this performance is achieved as the transmitted power vanishes. Through the wideband slope analysis, the increased energy requirements at low but nonzero power levels are determined. A similar analysis is also conducted in the wideband regime, and minimum bit energy and wideband slope expressions are obtained. In this regime, the required bit energy levels are found to be strictly greater than those achieved when Shannon capacity is considered. Overall, an energy-delay tradeoff is characterized.
\end{abstract}

\section{INTRODUCTION}

Next generation wireless systems will be designed to provide high-data-rate communications anytime, anywhere in a reliable and robust fashion while making efficient use of resources. This wireless vision will enable mobile multimedia communications. However, before this vision is realized, many technical challenges have to be addressed. In most wireless systems, spectral efficiency and energy efficiency are important considerations. Especially in mobile applications, energy resources are scarce and have to be conserved. Additionally, supporting quality of service (QoS) guarantees is one of the key requirements in the development of next generation wireless communication networks. For instance, in real-time services like multimedia video conference and live broadcast of sporting events, the key QoS metric is delay. In such cases, information has to be communicated with minimal delay. Satisfying the QoS requirements is especially challenging in wireless systems because channel conditions and hence, for instance, the data rates at which reliable communication can be established, vary randomly over time due to mobility and changing environment.

\footnotetext{
${ }^{1}$ This work was supported in part by the NSF CAREER Grant CCF0546384
}

Information theoretic analysis of wireless channels provides the ultimate performance limits and identifies the most efficient use of resources. For instance, Verdú in [2] has determined the minimum bit energy required for reliable communications over a general class of channels, and studied the spectral efficiency-bit energy tradeoff in the wideband regime. This work has provided a quantitative analysis of the energy-bandwidth tradeoff. On the other hand, delay and QoS constraints are generally not addressed in informationtheoretic studies. In [3], Wu and Negi considered statistical QoS guarantees and defined the effective capacity as the maximum constant arrival rate that a given service process can support while satisfying QoS requirements. In [7] and [8], Tang and Zhang proposed power and rate adaptation techniques for maximizing the system throughput subject to a given delay-QoS constraint. Specifically, by incorporating the concept of effective capacity [3]-[6], they converted the original problem to the one aiming at maximizing the effective capacity, by which the delay-QoS constraint is characterized by the QoS exponent $\theta$.

In this paper, we study the energy efficiency, which is of paramount importance in many wireless systems, in the presence of delay-QoS service constraints. Effective capacity can be seen as the maximum throughput that can be achieved by the given energy levels while providing statistical QoS guarantees. To identify the energy efficiency, we consider the bit energy which is defined as the average energy normalized by the effective capacity. We investigate the energy efficiency in the low-power and wideband regimes. Through this analysis, we quantify the increased energy requirements when the system is subject to statistical delay-QoS constraints.

\section{SySTEM MODEL}

We consider a point-to-point communication system in which there is one source and one destination. The general system model is depicted in Fig.1, and is similar to the one studied in [7]. In this model, it is assumed that the source generates data sequences which are divided into frames of duration $T$. These data frames are initially stored in the buffer before they are transmitted over the wireless channel. The discrete-time channel input-output relation in the $i^{\text {th }}$ symbol duration is given by

$$
y[i]=h[i] x[i]+n[i] \quad i=1,2, \ldots
$$




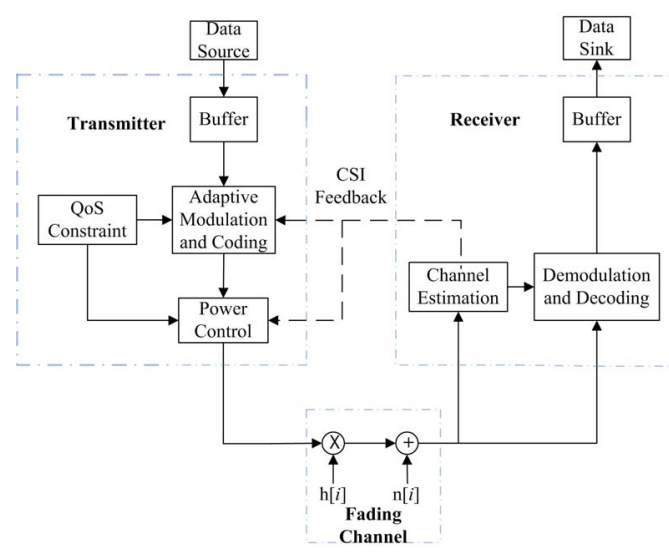

Fig. 1. The system model

where $x[i]$ and $y[i]$ denote the complex-valued channel input and output, respectively. The channel input is subject to an average power constraint $\mathbb{E}\left\{|x[i]|^{2}\right\} \leq \bar{P}$ for all $i$, and we assume that the bandwidth available in the system is $B$. Above, $n[i]$ is a zero-mean, circularly symmetric, complex Gaussian random variable with variance $\mathbb{E}\left\{|n[i]|^{2}\right\}=N_{0}$. The additive Gaussian noise samples $\{n[i]\}$ are assumed to form an independent and identically distributed (i.i.d.) sequence. Finally, $h[i]$ denotes the channel fading coefficient, and $\{h[i]\}$ is a stationary and ergodic discrete-time process. We assume that perfect channel state information (CSI) is available at the receiver while the transmitter has either no or perfect CSI. The availability of CSI at the transmitter is facilitated through CSI feedback from the receiver. Note that if the transmitter knows the channel fading coefficients, it employs power and rate adaptation. Otherwise, the signals are sent with constant power.

In the above system model, the average transmitted signalto-noise ratio is SNR $=\bar{P} /\left(N_{0} B\right)$. We denote the magnitudesquare of the fading coefficients by $z[i]=|h[i]|^{2}$. When there is only receiver CSI, instantaneous transmitted power is $P[i]=$ $\bar{P}$ and the instantaneous received SNR is expressed as $\gamma[i]=$ $\bar{P} z[i] /\left(N_{0} B\right)$. Moreover, the maximum instantaneous service rate $R[i]$ is

$$
R[i]=B \log _{2}(1+\mathrm{SNR} z[i]) \quad \text { bits/s. }
$$

When also the transmitter has CSI, the instantaneous service rate is

$$
R[i]=B \log _{2}\left(1+\mu_{\mathrm{opt}}(\theta, z[i]) z[i]\right) \quad \text { bits/s }
$$

where $\mu_{\text {opt }}(\theta, z)$ is the optimal power-adaptation policy. The power policy that maximizes the effective capacity, which will be discussed in Section III-A, is determined in [7]:

$$
\mu_{\mathrm{opt}}(\theta, z)= \begin{cases}\frac{1}{\alpha^{\frac{1}{\beta+1}} z^{\frac{\beta}{\beta+1}}}-\frac{1}{z} & z \geq \alpha \\ 0 & z<\alpha\end{cases}
$$

where $\beta=\frac{\theta T B}{\log _{e} 2}$ is the normalized QoS exponent and $\alpha$ is the channel threshold chosen to satisfy the average power constraint:

$$
\mathrm{SNR}=\mathbb{E}\left\{\mu_{\mathrm{opt}}(\theta, z)\right\}=\mathbb{E}\left\{\left[\frac{1}{\alpha^{\frac{1}{\beta+1}} z^{\frac{\beta}{\beta+1}}}-\frac{1}{z}\right] \tau(\alpha)\right\}
$$

where $\tau(\alpha)=1\{z \geq \alpha\}$ is the indicator function.

\section{EFFECTIVE CAPACITY AND SPECTRAL EFFICIENCY-BIT ENERGY TRADEOFF}

In this section, we briefly explain the notion of effective capacity and also describe the spectral efficiency-bit energy tradeoff. We refer the reader to [3] and [4] for more detailed exposition of the effective capacity.

\section{A. Effective Capacity}

In [3], Wu and Negi defined the effective capacity as the maximum constant arrival rate that a given service process can support in order to guarantee a QoS requirement specified by $\theta$. For time-varying arrival rates, effective capacity specifies the effective bandwidth of the arrival process that can be supported by the channel. If we define $Q$ as the stationary queue length, then $\theta$ is the decay rate of the tail distribution of the queue length:

$$
\lim _{q \rightarrow \infty} \frac{\log P(Q \geq q)}{q}=-\theta .
$$

Therefore, for large $q_{\max }$, we have the following approximation for the buffer violation probability: $P\left(Q \geq q_{\max }\right) \approx$ $e^{-\theta q_{\max }}$. Hence, while larger $\theta$ corresponds to more strict QoS constraints, smaller $\theta$ implies looser QoS guarantees. Similarly, if $D$ denotes the steady-state delay experienced in the buffer, then $P\left(D \geq d_{\max }\right) \approx e^{-\theta \delta d_{\max }}$ for large $d_{\max }$, where $\delta$ is determined by the arrival and service processes [9].

Let $\{R[i], i=1,2, \ldots\}$ denote the discrete-time stationary and ergodic stochastic service process and $S[t] \triangleq \sum_{i=1}^{t} R[i]$ be the time-accumulated process. Assume that the GärtnerEllis limit of $S[t]$, expressed as [10]

$$
\Lambda_{C}(\theta)=\lim _{t \rightarrow \infty} \frac{1}{t} \log _{e} \mathbb{E}\left\{e^{\theta S[t]}\right\}
$$

exists. Then, the effective capacity is defined as [3]

$$
C_{E}(\mathrm{SNR}, \theta)=-\frac{\Lambda_{C}(-\theta)}{\theta}=-\lim _{t \rightarrow \infty} \frac{1}{\theta t} \log _{e} \mathbb{E}\left\{e^{-\theta S[t]}\right\} .
$$

If the fading process $\{h[i]\}$ is constant during the frame duration $T$ and changes independently from frame to frame, then the effective capacity simplifies to

$$
C_{E}(\mathrm{SNR}, \theta)=-\frac{1}{\theta T} \log _{e} \mathbb{E}\left\{e^{-\theta T R[i]}\right\} \quad \text { bits/s }
$$

This block-fading assumption is an approximation for practical wireless channels, and the independence assumption can be justified if, for instance, transmitted frames are interleaved before transmission, or time-division multiple access is employed and frame duration is proportional to the coherence time of the channel. Throughout the paper, we denote the normalized 
effective capacity or equivalently the spectral efficiency in bits per second per Hertz by

$$
\mathrm{C}_{E}(\mathrm{SNR}, \theta)=\frac{C_{E}(\mathrm{SNR}, \theta)}{B}=-\frac{1}{\theta T B} \log _{e} \mathbb{E}\left\{e^{-\theta T R[i]}\right\}
$$

Note that a smaller $\theta$ means a higher delay violation probability of a specific delay bound, which implies a looser QoS guarantee. In particular, as $\theta$ approaches to 0 , there is no constraint on queue length and delay bound, and hence effective capacity converges to the Shannon capacity.

\section{B. Spectral Efficiency vs. Bit Energy}

In [2], Verdú has extensively studied the spectral efficiencybit energy tradeoff in the wideband regime. In this work, the minimum bit energy required for reliable communication over a general class of multiple-input multiple-output channels is identified. In general, if the capacity is a concave function of SNR, then the minimum bit energy is achieved as SNR $\rightarrow 0$. Verdú has also defined the wideband slope, which is the slope of the spectral efficiency curve at zero spectral efficiency. Wideband slope has emerged as a tool that enables us to analyze the energy efficiency at low but nonzero power levels and at large but finite bandwidths. In [2], the tradeoff between spectral efficiency and energy efficiency is analyzed considering the Shannon capacity. In this paper, we perform a similar analysis employing the effective capacity. Hence, we characterize the spectral efficiency-bit energy tradeoff under QoS constraints. We first have the following preliminary result.

Lemma 1: The normalized effective capacity, $C_{E}(\mathrm{SNR})$, given in (10) is a concave function of SNR.

Then, it can be easily seen that $\frac{E_{b}}{N_{0} \text { min }}$ under QoS constraints can be obtained from [2]

$$
\frac{E_{b}}{N_{0} \text { min }}=\lim _{\mathrm{SNR} \rightarrow 0} \frac{\mathrm{SNR}}{\mathrm{C}_{E}(\mathrm{SNR})}=\frac{1}{\dot{\mathrm{C}}_{E}(0)} .
$$

At $\frac{E_{b}}{N_{0}}$ min , the slope $\mathcal{S}_{0}$ of spectral efficiency versus $E_{b} / N_{0}$ is defined as [2]

$$
\mathcal{S}_{0}=\lim _{\frac{E_{b}}{N_{0}} \downarrow \frac{E_{b}}{N_{0}} \min _{\text {min }}} \frac{C_{E}\left(\frac{E_{b}}{N_{0}}\right)}{10 \log _{10} \frac{E_{b}}{N_{0}}-10 \log _{10} \frac{E_{b}}{N_{0}} \text { min }} 10 \log _{10} 2
$$

Considering the expression for normalized effective capacity, the wideband slope can be found from

$$
\mathcal{S}_{0}=-\frac{2\left(\dot{\mathrm{C}}_{E}(0)\right)^{2}}{\ddot{\mathrm{C}}_{E}(0)} \log _{e} 2
$$

where $\dot{C}_{E}(0)$ and $\ddot{C}_{E}(0)$ are the first and second derivatives, respectively, of the function $\mathrm{C}_{E}(\mathrm{SNR})$ in bits/s/Hz at zero SNR [2].

\section{ENERGy EFFiciency In the Low-Power Regime}

As discussed in the previous section, the minimum bit energy is achieved as SNR $=\frac{\bar{P}}{N_{0} B} \rightarrow 0$, and hence energy efficiency improves if one operates in the low-power or highbandwidth regime. From the Shannon capacity perspective, similar performances are achieved in these two regimes, which therefore can be seen as equivalent. However, as we shall see in this paper, considering the effective capacity leads to different results at low power and high bandwidth levels. In this section, we consider the low-power regime for fixed bandwidth, $B$, and study the spectral efficiency vs. bit energy tradeoff by finding the minimum bit energy and the wideband slope.

\section{A. CSI at the Receiver Only}

We initially consider the case in which only the receiver knows the channel conditions. Substituting (2) into (9), we obtain the spectral efficiency given $\theta$ in terms of SNR:

$$
\begin{aligned}
C_{E}(\mathrm{SNR}) & =-\frac{1}{\theta T B} \log _{e} \mathbb{E}\left\{e^{-\theta T B \log _{2}(1+\mathrm{SNR} z)}\right\} \\
& =-\frac{1}{\theta T B} \log _{e} \mathbb{E}\left\{(1+\mathrm{SNR} z)^{-\beta}\right\}
\end{aligned}
$$

where again $\beta=\frac{\theta T B}{\log _{e} 2}$. Note that since the analysis is performed for fixed $\theta$ throughout the paper, we henceforth express the effective capacity only as a function of SNR to simplify the expressions. The following result provides the minimum bit energy and the wideband slope. In the remainder of the paper, proofs are omitted due to space limitations.

Theorem 1: When only the receiver knows CSI, we have

$$
\frac{E_{b}}{N_{0 \text { min }}}=\frac{\log _{e} 2}{\mathbb{E}\{z\}} \text { and } \mathcal{S}_{0}=\frac{2}{(\beta+1) \frac{\mathbb{E}\left\{z^{2}\right\}}{(\mathbb{E}\{z\})^{2}}-\beta} .
$$

From the above result, we immediately see that the minimum received bit energy is

$$
{\frac{E_{b}^{r}}{N_{0} \text { min }}}^{r}=\frac{E_{b}}{N_{0} \text { min }} \mathbb{E}\{z\}=\log _{e} 2=-1.59 \mathrm{~dB} .
$$

Note that if the Shannon capacity is used in the analysis, $\frac{E_{b}^{r}}{N_{0}}=-1.59 \mathrm{~dB}$ and $\mathcal{S}_{0}=2 /\left(\mathbb{E}\left\{z^{2}\right\} / \mathbb{E}^{2}\{z\}\right)$ Therefore, we conclude from Theorem 1 that as the power decreases, energy efficiency approaches the performance achieved by a system that does not have QoS limitations. However, we note that wideband slope is smaller if $\theta>0$. Hence, having QoS constraint decreases the spectral efficiency or equivalently increases the energy requirements for fixed spectral efficiency values at low but nonzero SNR levels.

Figure 2 plots the spectral efficiency as a function of the bit energy for different values of $\theta$ in the Rayleigh fading channel with $\mathbb{E}\left\{|h|^{2}\right\}=\mathbb{E}\{z\}=1$. Note that the curve for $\theta=0$ corresponds to the Shannon capacity. Throughout the paper, we set the frame duration to $T=2 \mathrm{~ms}$ in the numerical results. For the fixed bandwidth case, we have assumed $B=10^{5}$ Hz. In Fig. 2, we observe that all curves approach $\frac{E_{b}}{N_{0} \min }=$ $-1.59 \mathrm{~dB}$ as predicted. On the other hand, we note that the wideband slope decreases as $\theta$ increases. Therefore, at low but nonzero spectral efficiencies, more energy is required as the QoS constraints become more stringent.

\section{B. CSI at both the Transmitter and Receiver}

We now consider the case in which both the transmitter and receiver have CSI. Substituting (3) into (9), we have

$$
\begin{aligned}
C_{E}(\mathrm{SNR}) & =-\frac{1}{\theta T B} \log _{e} \mathbb{E}\left\{e^{-\theta T B \log _{2}\left(1+\mu_{\mathrm{opt}}(\theta, z) z\right)}\right\} \\
& =-\frac{1}{\theta T B} \log _{e}\left(F(\alpha)+\mathbb{E}\left\{\left(\frac{z}{\alpha}\right)^{-\frac{\beta}{\beta+1}} \tau(\alpha)\right\}\right)
\end{aligned}
$$




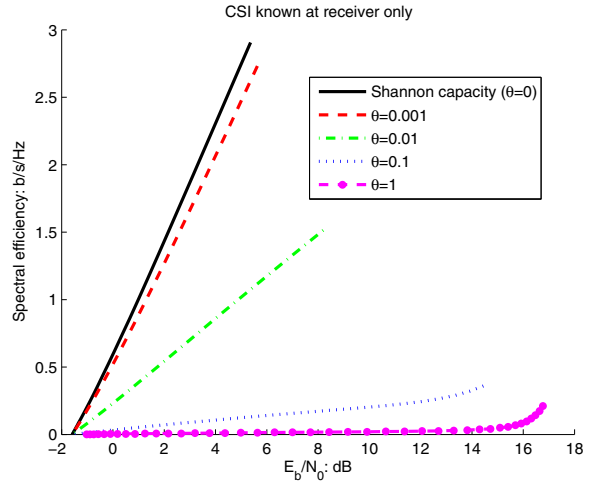

Fig. 2. Spectral efficiency vs. $E_{b} / N_{0}$ in the Rayleigh fading channel with fixed $B$; CSI known at receiver only

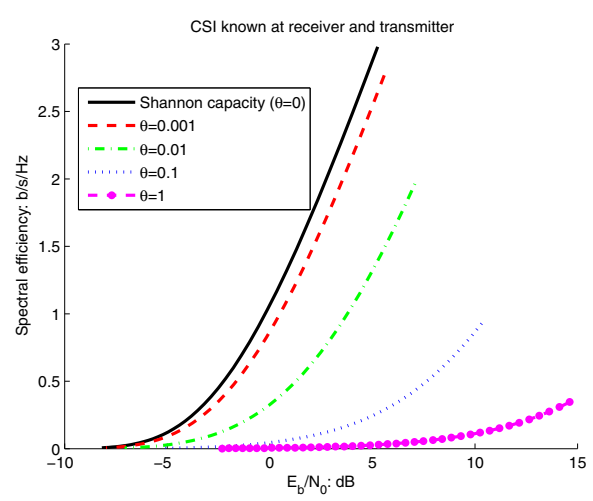

Fig. 3. Spectral efficiency vs. $E_{b} / N_{0}$ in the Rayleigh fading channel with fixed $B$; CSI known at transmitter and receiver

where $F(\alpha)=\mathbb{E}\{1\{z<\alpha\}\}$. For this case, following an approach similar to that in [11], we obtain the following result.

Theorem 2: When both the transmitter and receiver have CSI, the minimum bit energy with optimal power control and rate adaptation becomes

$$
{\frac{E_{b}}{N_{0 \text { min }}}}=\frac{\log _{e} 2}{z_{\max }}
$$

where $z_{\max }$ is the maximum value that the random variable $z$ can take.

Note that for distributions with unbounded support, $z_{\max }=\infty$ and hence $\frac{E_{b}}{N_{0}}$ min $=0=-\infty \mathrm{dB}$. In this case, it is easy to see that the wideband slope is $S_{0}=0$.

We note that as in the case in which there is CSI at the receiver, the minimum bit energy achieved under QoS constraints is the same as that achieved by the Shannon capacity [11]. Hence, the energy efficiency again approaches the performance of an unconstrained system as power diminishes.

Fig. 3 plots the spectral efficiency vs. bit energy for different values of $\theta$ in the Rayleigh fading channel with $\mathbb{E}\{z\}=1$. In all cases, we observe that the bit energy goes to $-\infty$ as the spectral efficiency decreases. We also note that at small but nonzero spectral efficiencies, the required energy is higher as $\theta$ increases.

\section{ENERGY EFFICIENCY IN THE WIDEBAND REgIME}

In this section, we study the performance at high bandwidths while the average power $\bar{P}$ is kept fixed. We investigate the impact of $\theta$ on $\frac{E_{b}}{N_{0} \min }$ and the wideband slope $\mathcal{S}_{0}$ in this wideband regime. Note that as the bandwidth increases, the average signal-to-noise ratio SNR $=\bar{P} /\left(N_{0} B\right)$ and the spectral efficiency decreases.

\section{A. CSI at the Receiver Only}

We define $\zeta=\frac{1}{B}$ and express the spectral efficiency (14) as a function of $\zeta$ :

$$
\mathrm{C}_{E}(\zeta)=-\frac{\zeta}{\theta T} \log _{e} \mathbb{E}\left\{e^{-\frac{\theta T}{\zeta} \log _{2}\left(1+\frac{\bar{P} \zeta}{N_{0}} z\right)}\right\} .
$$

The bit energy is again defined as

$$
\frac{E_{b}}{N_{0}}=\frac{\mathrm{SNR}}{\mathrm{C}_{E}(\mathrm{SNR})}=\frac{\bar{P} \zeta}{\mathrm{C}_{0}}=\frac{\bar{P}}{\mathrm{C}_{E}(\zeta)}=\frac{\mathrm{C}_{E}(\zeta) / \zeta}{.}
$$

It can be readily verified that $\mathrm{C}_{E}(\zeta) / \zeta$ monotonically increases as $\zeta \rightarrow 0$ (or equivalently $B \rightarrow \infty$ ). Therefore

$$
\frac{E_{b}}{N_{0} \min }=\lim _{\zeta \rightarrow 0} \frac{\bar{P} \zeta / N_{0}}{\mathrm{C}_{E}(\zeta)}=\frac{\bar{P} / N_{0}}{\dot{\mathrm{C}}_{E}(0)}
$$

where $\dot{C}_{E}(0)$ is the first derivative of the spectral efficiency with respect to $\zeta$ at $\zeta=0$. The wideband slope $\mathcal{S}_{0}$ can be obtained from the formula (13) by using the first and second derivatives of the spectral efficiency $C_{E}(\zeta)$ with respect to $\zeta$.

Theorem 3: When only the receiver has CSI, the minimum bit energy and wideband slope in the wideband regime are given by

$$
\begin{gathered}
E_{b}=\frac{-\frac{\theta T \bar{P}}{N_{0}}}{N_{0} \text { min }}=\frac{(22)}{\log _{e} \mathbb{E}\left\{e^{-\frac{\theta T \bar{P}}{N_{0} \log 2} z}\right\}} \\
\mathcal{S}_{0}=2\left(\frac{N_{0} \log _{e} 2}{\theta T \bar{P}}\right)^{2} \frac{\mathbb{E}\left\{e^{-\frac{\theta T \bar{P}}{N_{0} \log _{e} 2} z}\right\}\left(\log _{e} \mathbb{E}\left\{e^{-\frac{\theta T \bar{P}}{N_{0} \log _{e} 2} z}\right\}\right)^{2}}{\mathbb{E}\left\{e^{-\frac{\theta T \bar{P}}{N_{0} \log _{e} 2} z} z^{2}\right\}}
\end{gathered}
$$

It is interesting to note that unlike the low-power regime results, we now have

$$
\frac{E_{b}}{N_{0} \text { min }}=\frac{-\frac{\theta T \bar{P}}{N_{0}}}{\log _{e} \mathbb{E}\left\{e^{-\frac{\theta \theta \bar{P}}{N_{0} \log _{e} 2}}\right\}} \geq \frac{-\frac{\theta T \bar{P}}{N_{0}}}{\mathbb{E}\left\{\log _{e} e^{-\frac{\theta \theta \bar{P}}{N_{0} \log _{e} 2}}\right\}}=\frac{\log _{e} 2}{\mathbb{E}\{z\}}
$$

where Jensen's inequality is used. Therefore, we will be operating above $-1.59 \mathrm{~dB}$ unless there are no QoS constraints and hence $\theta=0$. For the Rayleigh channel, we can specialize (22) and (23) to obtain

$$
\begin{gathered}
{\frac{E b}{N_{0}}}_{N_{\text {min }}}=\frac{\frac{\theta T \bar{P}}{N_{0}}}{\log _{e}\left(1+\frac{\theta T \bar{P}}{N_{0} \log _{e} 2}\right)} \\
\mathcal{S}_{0}=\left(\frac{N_{0} \log _{e} 2}{\theta T \bar{P}} \log _{e}\left(1+\frac{\theta T \bar{P}}{N_{0} \log _{e} 2}\right)+\log _{e}\left(1+\frac{\theta T \bar{P}}{N_{0} \log _{e} 2}\right)\right)^{2}
\end{gathered}
$$

It can be easily seen that in the Rayleigh channel, the minimum bit energy monotonically increases with increasing $\theta$. Fig. 4 plots the spectral efficiency curves as a function of bit energy 


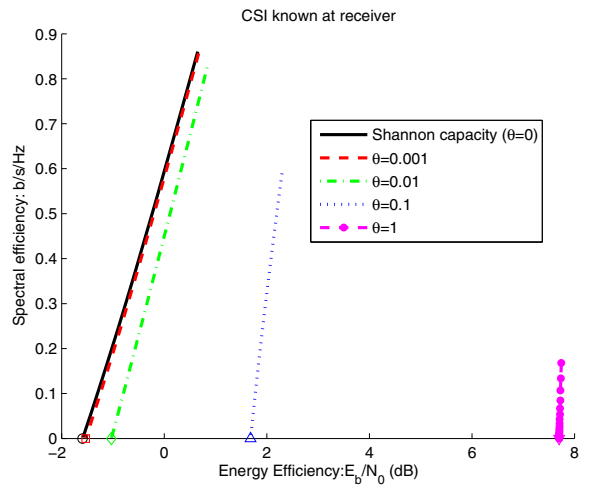

Fig. 4. Spectral efficiency vs. $E_{b} / N_{0}$ in the Rayleigh fading channel with fixed $\bar{P}$; CSI known at receiver only

in the Rayleigh channel. In all the curves, we set $\bar{P} / N_{0}=10^{4}$. We immediately observe that more stringent QoS constraints and hence higher values of $\theta$ lead to higher minimum bit energy values and also higher energy requirements at other nonzero spectral efficiencies. The wideband slope values are found to be equal to $\mathcal{S}_{0}=\{1.0288,1.2817,3.3401,12.3484\}$ for $\theta=\{0.001,0.01,0.1,1\}$, respectively.

\section{B. CSI at both the Transmitter and Receiver}

To analyze $\frac{E_{b}}{N_{0}}$ in this case, we initially obtain the following result and identify the limiting value of the threshold $\alpha$ as the bandwidth increases to infinity.

Theorem 4: In wideband regime, the threshold $\alpha$ in the optimal power adaptation scheme converges to

$$
\lim _{\zeta \rightarrow 0} \alpha(\zeta)=\alpha^{*}
$$

where $\alpha^{*}$ satisfies

$$
\mathbb{E}\left\{\left[\frac{\log _{e}\left(\frac{z}{\alpha^{*}}\right)}{z}\right] \tau\left(\alpha^{*}\right)\right\}=\frac{\theta T \bar{P}}{N_{0} \log _{e} 2}
$$

Moreover, for $\theta>0, \alpha^{*}<\infty$.

The spectral efficiency in this case is given by

$$
\mathrm{C}_{E}(\zeta)=-\frac{\zeta}{\theta T} \log _{e}\left(F(\alpha)+\mathbb{E}\left\{\left(\frac{z}{\alpha}\right)^{-\frac{\theta T}{\theta T+\zeta \log _{e} 2}} \tau(\alpha)\right\}\right)
$$

where again $F(\alpha)=\mathbb{E}\{1\{z<\alpha\}\}$ and $\tau(\alpha)=1\{\tau \geq \alpha\}$. The following result provides the minimum bit energy and wideband slope.

Theorem 5: When both the receiver and transmitter have CSI, the bit energy required at zero spectral efficiency and wideband slope in the wideband regime are given by

$$
\frac{E_{b}}{N_{0} \text { min }}=-\frac{\theta T \bar{P}}{N_{0} \log _{e} \xi} \text { and } \mathcal{S}_{0}=\frac{\xi\left(\log _{e} \xi\right)^{2} \log _{e} 2}{\theta T\left(\frac{\bar{P} \alpha^{*}}{N_{0}}+\dot{\alpha}(0) \mathbb{E}\left\{\frac{1}{z} \tau\left(\alpha^{*}\right)\right\}\right)}
$$

where $\xi=F\left(\alpha^{*}\right)+\mathbb{E}\left\{\frac{\alpha^{*}}{z} \tau\left(\alpha^{*}\right)\right\}$, and $\dot{\alpha}(0)$ is the derivative of $\alpha$ with respect to $\zeta$, evaluated at $\zeta=0$.

It is interesting to note that the minimum bit energy is strictly greater than zero for $\theta>0$. Hence, we see a

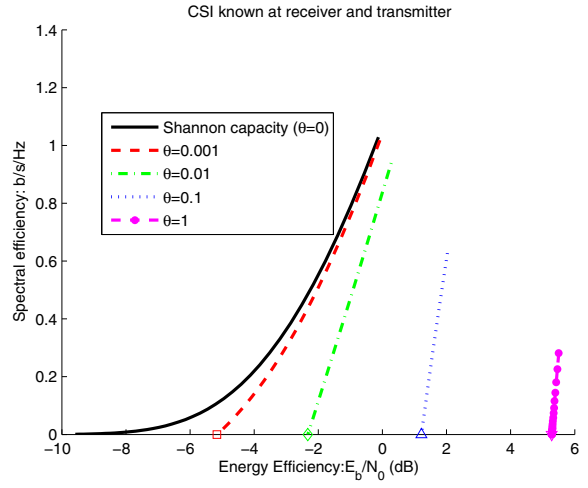

Fig. 5. Spectral efficiency vs. $E_{b} / N_{0}$ in the Rayleigh fading channel with fixed $\bar{P}$; CSI known at transmitter and receiver

stark difference between the wideband regime and low-power regime in which the minimum bit energy is zero for fading distributions with unbounded support. Fig. 5 plots the spectral efficiency curves in the Rayleigh fading channel and is in perfect agreement with the theoretical results. Obviously, the plots are drastically different from those in the low-power regime where all curves approach $-\infty$ as the spectral efficiency decreases. In Fig. 5, the minimum bit energy is finite for the cases in which $\theta>0$. Generally speaking, because of power and rate adaptation, $\frac{E_{b}}{N_{0}}$ min in this case is smaller compared with the case in which only the receiver has CSI (see Fig. 4). The wideband slope values are computed to be equal to $\mathcal{S}_{0}=\{0.3081,1.0455,2.5758,4.1869\}$.

\section{REFERENCES}

[1] T. S. Rappaport, "Wireless Communications: Principles and Practice", 2nd ed. Prentice Hall PTR, 2001.

[2] S. Verdú, "Spectral efficiency in the wideband regime," IEEE Trans. Inform. Theory, vol.48, no.6 pp.1319-1343. Jun.2002.

[3] D. Wu and R. Negi "Effective capacity: a wireless link model for support of quality of service," IEEE Trans. Wireless Commun., vol.2,no. 4, pp.630-643. July 2003.

[4] D. Wu and R. Negi, "Effective capacity-based quality of service measures for wireless networks," Broadband Networks,2004.BroadNets 2004. Proc. First International Conference on. pp. 527-536.

[5] D. Wu and R. Negi, "Downlink scheduling in a cellular network for quality-of-service assurance," IEEE Trans. Veh. Technol., vol.53, no.5, pp. 1547-1557, Sep., 2004.

[6] D. Wu and R. Negi, "Utilizing multiuser diversity for efficient support of quality of service over a fading channel," IEEE Trans. Veh. Technol., vol.49, pp. 1073-1096, May 2003.

[7] J. Tang and $X$. Zhang, "Quality-of-service driven power and rate adaptation over wireless links," IEEE Trans. Wireless Commun., vol. 6, no. 8, pp.3058-3068, Aug. 2007.

[8] J. Tang and X. Zhang, "Quality-of-service driven power and rate adaptation for multichannel communications over wireless links," IEEE Trans. Wireless Commun., vol. 6, no. 12, pp.4349-4360, Dec. 2007.

[9] J. Tang and X. Zhang, "Cross-layer-model based adaptive resource allocation for statistical QoS guarantees in mobile wireless networks," IEEE Trans. Wireless Commun., vol. 7, pp.2318-2328, June 2008.

[10] C.-S. Chang, "Stability, queue length, and delay of deterministic and stochastic queuing networks," IEEE Trans. Auto. Control, vol. 39, no. 5, pp. 913-931, May 1994.

[11] S. Shamai, S. Verdú, "The impact of frequency-flat fading on the spectral efficiency of CDMA," IEEE Trans. Inform. Theory, vol. 47, no. 4, pp. 1302-1327, May 2001. 